\title{
Transplantation of spermatogonial stem cells isolated from leukemic mice restores fertility without inducing leukemia
}

\author{
Kazutoshi Fujita, ${ }^{1}$ Hiroshi Ohta, ${ }^{2}$ Akira Tsujimura, ${ }^{1}$ Tetsuya Takao, ${ }^{1}$ Yasushi Miyagawa, ${ }^{1}$ \\ Shingo Takada, ${ }^{1}$ Kiyomi Matsumiya, ${ }^{1}$ Teruhiko Wakayama, ${ }^{2}$ and Akihiko Okuyama ${ }^{1}$ \\ 1Department of Urology, Osaka University Graduate School of Medicine, Osaka, Japan. 'Laboratory for Genomic Reprogramming, \\ Center for Developmental Biology, Institute of Physical and Chemical Research (RIKEN), Kobe, Japan.
}

\begin{abstract}
More than $\mathbf{7 0} \%$ of patients survive childhood leukemia, but chemotherapy and radiation therapy cause irreversible impairment of spermatogenesis. Although autotransplantation of germ cells holds promise for restoring fertility, contamination by leukemic cells may induce relapse. In this study, we isolated germ cells from leukemic mice by FACS sorting. The cell population in the high forward-scatter and low side-scatter regions of dissociated testicular cells from leukemic mice were analyzed by staining for $\mathrm{MHC}$ class I heavy chain $\left(\mathrm{H}-2 \mathrm{~K}^{\mathrm{b}}\right.$ / $\left.H-2 D^{b}\right)$ and for CD45. Cells that did not stain positively for $H-2 K^{b} / H-2 D^{b}$ and CD45 were sorted as the germ cell-enriched fraction. The sorted germ cell-enriched fractions were transplanted into the testes of recipient mice exposed to alkylating agents. Transplanted germ cells colonized, and recipient mice survived. Normal progeny were produced by intracytoplasmic injection of sperm obtained from recipient testes. When unsorted germ cells from leukemic mice were transplanted into recipient testes, all recipient mice developed leukemia. The successful birth of offspring from recipient mice without transmission of leukemia to the recipients indicates the potential of autotransplantation of germ cells sorted by FACS to treat infertility secondary to anticancer treatment for childhood leukemia.
\end{abstract}

\section{Introduction}

The incidence of childhood cancer is approximately 141 per million annually, and leukemia is the most common childhood cancer, accounting for approximately $30 \%$ of cancers diagnosed in children less than 15 years of age (1). Due to the development of treatment modalities such as chemotherapy, more than $70 \%$ of patients survive (2) and therefore require long-term follow-up of late side effects. Although chemotherapy and radiation therapy provide great advantages for patients with cancer, they cause irreversible impairment of spermatogenesis. Because $23-30 \%$ of survivors show azoospermia $(3,4)$, infertility has become an important quality of life issue (5). With assisted reproductive techniques, adult men with cancer have a chance to have biological children by ejaculation and cryopreservation of their sperm before treatment (6) even if they develop irreversible azoospermia after chemotherapy. Because the mature germ cells necessary for intracytoplasmic sperm injection do not develop until the onset of puberty (7), prepubertal patients who have only spermatogonia and spermatocytes in their testes do not benefit from cryopreservation of their sperm and assisted reproductive techniques. To date, the only established clinical option for preservation of fertility is cryopreservation of sperm. Recently, a new technique was developed for germ cell transplantation in mice $(8,9)$. Donor germ cells, including spermatogonial stem cells, are injected into the seminiferous tubules of recipient mice; the transplanted stem cells undergo spermatogenesis, and recipient mice

Nonstandard abbreviations used: CZB, Chatot-Ziomek-Bavister; FSC, forward scatter; PI, propidium iodide; SSC, side scatter.

Conflict of interest: Osaka University and RIKEN have submitted a patent on the isolation of germ cells. K. Fujita, H. Ohta, A. Tsujimura, K. Matsumiya, and A. Okuyama were listed as co-inventors.

Citation for this article: J. Clin. Invest. 115:1855-1861 (2005). doi:10.1172/JCI24189. transmit the donor haplotype to their progeny. Transplantation of germ cells has been successful even in primates (10).

This technique may provide for preservation of fertility in prepubertal patients. Before chemotherapy, germ cells, including spermatogonial stem cells, may be harvested and cryopreserved. After the patient recovers from cancer and undergoes puberty, germ cells can be autotransplanted and fertility thereby restored. However, there is a risk of contamination of harvested germ cells by malignant cells. Jahnukainen et al. reported that transplantation of testicular cells from leukemic rats induces transmission of leukemia, indicating that this techniques should not be used in humans (11). Contamination of donor samples by leukemic cells may lead to relapse. Careful isolation of germ cells, including stem cells, from malignant cells is necessary prior to transplantation. Development of a procedure to isolate testicular germ cells and avoid contamination remains one of the greatest challenges to the clinical application of autotransplantation to infertility treatment.

We developed a procedure to accurately isolate germ cells from leukemic mice by FACS with antibodies against 2 surface markers, MHC class I and common leukocyte antigen (CD45). We performed germ cell transplantation and showed successful development of germ cells to mature sperm in testes rendered azoospermic by chemotherapy.

\section{Results}

Validation of surface markers for the isolation of germ cells and leukemic cells. There are requirements for the selection of surface markers for isolation of germ cells from leukemic cells by FACS. First, the donor cell population should not contain any cancer cells. Second, isolated germ cells must include spermatogonial stem cells that can undergo spermatogenesis after transplantation. We investigated the validity of 2 groups of surface markers for isolation: first, CD45, CD13, and CD69 as surface markers for leukemic cells; and second, 

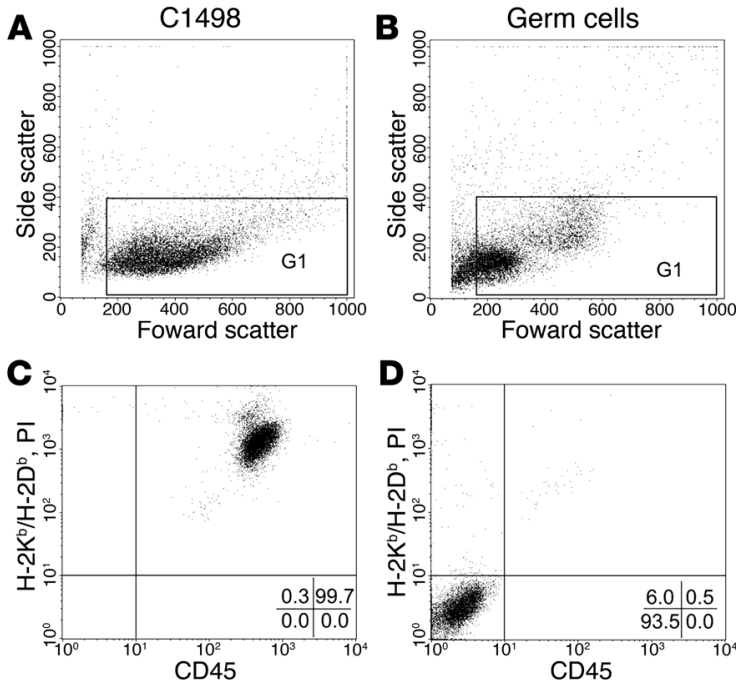

Figure 1

Flow cytometric analysis of $\mathbf{C} 1498$ cells $(\mathbf{A}$ and $\mathbf{C})$ and germ cells (B and D) from normal C57BL/6 mice. Gates for FACS were set for FSC and SSC (A and B). G1 comprises the FSC ${ }^{\text {high }}$ and SSClow fractions. The staining profiles of $C D 45$ compared with $\mathrm{H}-2 \mathrm{~K}^{\mathrm{b}} / \mathrm{H}-2 \mathrm{D}^{\mathrm{b}}$ for the $\mathrm{G} 1$ cell fraction of C1498 cells (C) and germ cells (D) from normal C57BL/6 mice are shown with quadrant statistics. None of the $\mathrm{C} 1498$ cells in the $\mathrm{FSC}$ high and $\mathrm{SSC}^{\text {low }}$ fractions were in the $\mathrm{CD} 45^{-}$and $\mathrm{H}-2 \mathrm{~K}^{\mathrm{b}} / \mathrm{H}-2 \mathrm{D}^{\mathrm{b}-}$ fraction, whereas $93.5 \%$ of the germ cells were in the $\mathrm{CD}^{4} 5^{-}$and $\mathrm{H}-2 \mathrm{~K}^{\mathrm{b}} / \mathrm{H}-2 \mathrm{D}^{\mathrm{b}-}$ fraction.

MHC class I, c-kit, and Thy-1.2 as surface markers for spermatogonial stem cells. We used the $\mathrm{C} 1498$ cell line, a murine leukemia cell line of C57BL/ 6 origin, and dissociated cells from normal C57BL/ 6 mouse testes obtained at 5 weeks of age. Flow cytometric analysis showed that 2 antibodies, 1 against CD45 and 1 against MHC class I heavy chain $\left(\mathrm{H}-2 \mathrm{~K}^{\mathrm{b}} / \mathrm{H}-2 \mathrm{D}^{\mathrm{b}}\right)$, could be used to successfully differentiate germ cells from leukemic cells (Figure 1). Dissociated germ cells and C1498 cells clustered in a similar area of a bivariate histogram of forward scatter (FSC) and side scatter (SSC) (Figure $1, A$ and B). In the FSChigh and SSClow cell population area (G1), $99.7 \%$ of the $\mathrm{C} 1498$ cells stained positively for both the anti-H- $2 \mathrm{~K}^{\mathrm{b}} /$ $\mathrm{H}-2 \mathrm{D}^{\mathrm{b}}$ and the anti-CD45 antibodies. There were no C1498 cells in the $\mathrm{H}-2 \mathrm{~K}^{\mathrm{b}} / \mathrm{H}-2 \mathrm{D}^{\mathrm{b}-}$ and $\mathrm{CD} 45^{-}$fractions, whereas $93.5 \%$ germ cells were observed in the $\mathrm{CD}^{4} 5^{-}$and $\mathrm{H}-2 \mathrm{~K}^{\mathrm{b}} / \mathrm{H}-2 \mathrm{D}^{\mathrm{b}-}$ fraction (Figure $1, \mathrm{C}$ and $\mathrm{D}$ ). Cells of the $\mathrm{H}-2 \mathrm{~K}^{\mathrm{b}} / \mathrm{H}-2 \mathrm{D}^{\mathrm{b}-}$ fraction were confirmed as being germ cells by positive immunostaining with TRA98, a monoclonal antibody that recognizes the nucleus of spermatogonia, spermatocytes, and spermatids but does not recognize elongated spermatids, mature spermatozoa, or somatic cells (12) (Figure 2).

Sorting of germ cells from C1498-inoculated C57BL/6 mice. We set the gate containing the FSC ${ }^{\text {high }}$ and SSClow region (G1) as an initial gating parameter (Figure 3A). Cells in the G1 fraction were analyzed by staining for $\mathrm{H}-2 \mathrm{~K}^{\mathrm{b}} / \mathrm{H}-2 \mathrm{D}^{\mathrm{b}}$ and $\mathrm{CD} 45$, and we set the gates for the $\mathrm{H}-2 \mathrm{~K}^{\mathrm{b}} / \mathrm{H}-2 \mathrm{D}^{\mathrm{b}-}$ and $\mathrm{CD} 45^{-}$regions $(\mathrm{G} 2)$ as the germ cell-enriched fractions and the gates for the $\mathrm{H}-2 \mathrm{~K}^{\mathrm{b}} / \mathrm{H}-2 \mathrm{D}^{\mathrm{b}+}$ and $\mathrm{CD} 45^{+}$regions (G3) as the leukemic cell fractions (Figure 3B). G1, G2, and G3 were set so that no C1498 cells stained with both antibodies fell into the G2 region. The G2 fraction represented $51.4 \%$ of the total cells, and the G3 fraction, which consisted of normal leukocyte and leukemic cells, represented $2.5 \%$ of the total cells. The G2 and G3 fractions were isolated by FACS from dissociated cells from C1498-inoculated mice and injected intraperitoneally into recipient mice. All 12 mice injected with the germ cell-enriched fractions (G2) survived without onset of leukemia for 300 days whereas all 12 mice injected with the leukemic cell fractions (G3) developed typical signs of terminal leukemia with hemorrhagic ascites within 40 days (Figure 3C). Histologic analysis of bone marrow and peritoneal exudates from surviving mice showed no signs of leukemia (Figure 4).

Transplantation of the germ cell-enriched fraction from C1498-inoculated GFP mice. We examined the capacity of sorted germ cells to undergo spermatogenesis in recipient testes treated with busulfan; this capacity would indicate that spermatogonial stem cells were included among the sorted germ cells. GFP mice were used as donor mice to allow for differentiation between recipient cells and transplanted germ cells. Because dissociated cells from GFP mice fell into the FITC-positive region, dissociated cells from 8-week-old C1498-inoculated GFP mice were stained with biotin-conjugated anti- $\mathrm{H}-2 \mathrm{~K}^{\mathrm{b}} /$ $\mathrm{H}-2 \mathrm{D}^{\mathrm{b}}$, PE-conjugated streptavidin, and PE-Cy5-conjugated antiCD45 instead of FITC-conjugated anti-CD45. Gates (G1 and G2) were set as described above, and the germ cell-enriched fraction (G2) was isolated. The viability of cells in the isolated germ cell-enriched fraction was more than $95 \%$ as measured by trypan blue exclusion. The sorted germ cell-enriched fraction was transplanted into the seminiferous tubules of 8-week-old recipient mice via injection into efferent ducts. Recipient mice were pretreated with busulfan to eliminate endogenous spermatogenesis 4 weeks prior to transplantation. Eight weeks after transplantation, all recipient mice remained alive (Figure 5). When unsorted dissociated germ cells from C1498-inoculated $\mathrm{C} 57 \mathrm{BL} / 6$ mice were transplanted into recipient testes, all recipient mice developed typical signs of terminal leukemia within 4 weeks after transplantation. Cross-sectioning of a testis transplanted with unsorted germ cells at 2 weeks after transplantation showed infiltration by leukemic cells (Figure 6, A and B). Dissected testes transplanted with sorted germ cells had a normal appearance, similar to that of testes treated with busulfan. GFP donor cells were observed in the seminiferous tubules of 11 of 12 testes of 6 recipient mice (Figure 6, C-F). In seminiferous tubules containing GFP donor cells, normal spermatogenesis and mature sperm were observed (Figure 6, G-I). Histologic analysis of recipient testes showed no leukemic cells. Thus, the sorted germ cell-enriched fraction contained a sufficient number of spermatogonial stem cells to provide for spermatogenesis, and no contamination by leukemic cells occurred.

To investigate whether the GFP sperm obtained from recipient testes had the ability to support normal development, intracyto-
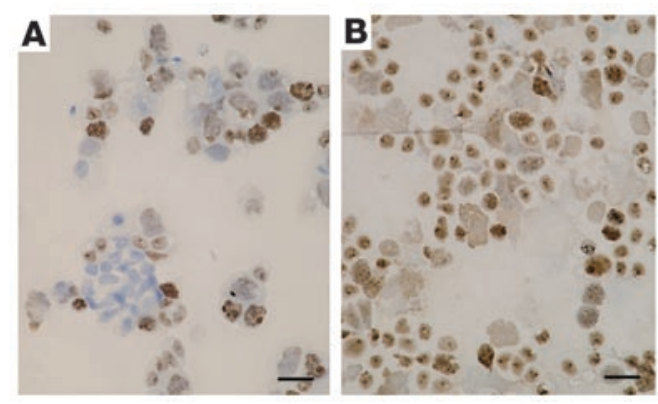

\section{Figure 2}

Immunostaining of germ cells before isolation $(\mathbf{A})$ and of the germ cell-enriched fraction (B) with the TRA98 monoclonal antibody specific for nuclei of germ cells from spermatogonia to spermatids. After isolation, the germ cell-enriched fraction only consisted of germ cells. Scale bars: $20 \mu \mathrm{m}$. 

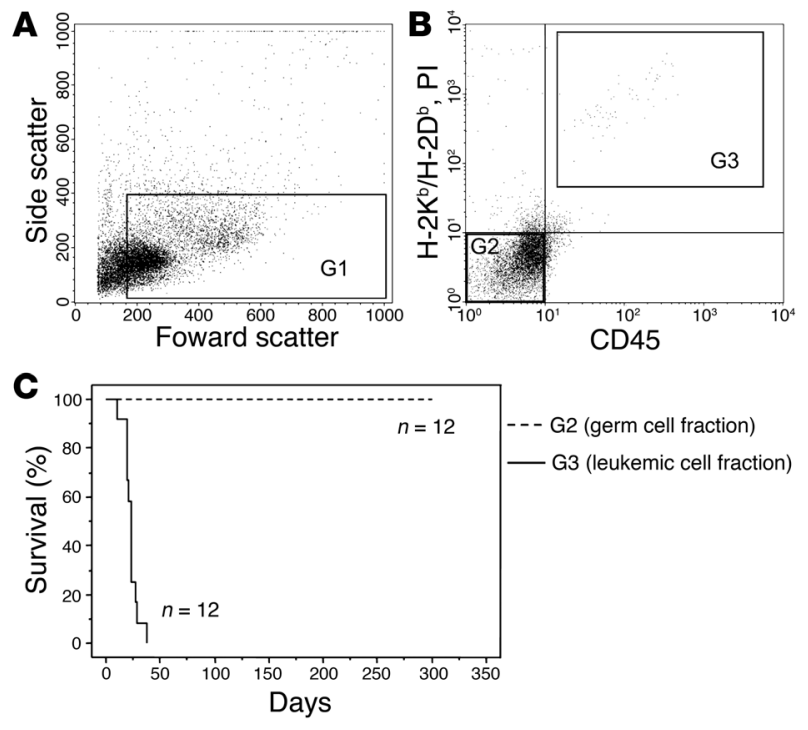

plasmic sperm injections were performed. Of the 42 embryos constructed, 40 (95.2\%) developed to the 2-cell stage within 24 hours of culture. After transfer of the embryos into the oviducts of pseudopregnant female mice, a total of 12 live pups (28.6\%) were born (Figure 7). Because haploid spermatids can share cytoplasm, and thus a GFP sperm cell picked for fertilization may not transmit the GFP transgene to progeny, approximately half of the pups (5 pups) showed green fluorescence under excitation light, indicating that sperm were derived from transplanted germ cells.

\section{Discussion}

To date, the only established clinical option for preservation of fertility is cryopreservation of sperm. There is no clinical technique currently available to preserve fertility in the prepubertal boy who is likely to be sterilized by chemotherapy or radiotherapy, because prepubertal boys have no mature sperm. To overcome this problem, testicular tissues could be harvested before treatment from prepubertal patients who have only spermatogonia and spermatocytes, then cryopreserved; immature germ cells could somehow be matured either by autotransplantation, in vitro maturation, or xenografting. Because the seminiferous tubules of testes contain functional Sertoli cells even after chemotherapy or radiation $(10,13,14)$, autotransplantation into seminiferous tubules would mature immature germ cells to become sperm. However, there are at least 3 hurdles to overcome before this idea can be applied clinically. The first is that all malignant cells must be excluded from germ cells to be transplanted because contamination by one malignant cell may lead to relapse $(11,15)$. Second, dissected testicular tissue may contain only

\section{Figure 4}

Histologic analysis of intraperitoneally injected mice. Histologic analysis of mice injected with the germ cell-enriched fraction that survived for 300 days showed no signs of leukemia (A, D, and $\mathbf{G})$ whereas histologic analysis of mice injected with the leukemic cell fraction showed infiltration of leukemic cells to the bone marrow (B and $\mathbf{E})$; hemorrhagic ascites also contained leukemic cells (H). (C, F, and I) Control mice. (A-C) H\&E-stained bone marrow. (D-F) May-Giemsa-stained bone marrow. (G-I) May-Giemsa-stained peritoneal exudates. Scale bars: $100 \mu \mathrm{m}$ (A-C); $20 \mu \mathrm{m}$ (D-I).

\section{Figure 3}

Isolation of the germ cell-enriched fraction and the leukemic fraction from leukemic mice. (A and B) Flow cytometric analysis of the G1 fraction of germ cells from $\mathrm{C} 1498$-inoculated mice. The $\mathrm{H}-2 \mathrm{~K}^{\mathrm{b}} / \mathrm{H}-2 \mathrm{D}^{\mathrm{b}-}$ and CD45- fraction composes the germ cell-enriched fraction (G2), and the $\mathrm{H}-2 \mathrm{~K}^{\mathrm{b}} / \mathrm{H}-2 \mathrm{D}^{\mathrm{b}+}$ and $\mathrm{CD} 45^{+}$fraction composes the leukemic cell fraction (G3). Each fraction (G2 and G3) was isolated by FACS. (C) Survival after intraperitoneal injection of cell fractions sorted by FACS. All mice injected with the germ cell-enriched fraction (G2) survived without onset of leukemia for 300 days $(n=12)$ whereas all mice injected with the leukemic cell fraction (G3) developed terminal signs of leukemia within 40 days $(n=12)$.

a few germ cells due to infiltration of malignant cells, but dissection of a large amount of tissue for autotransplantation should be avoided, and one testis should be preserved as a recipient. Thus, we must be able to induce in vitro proliferation of a few spermatogonial stem cells from a small number of dissected seminiferous tubules. Third, isolated immature germ cells must be able to undergo normal spermatogenesis by means of autotransplantation and, furthermore, the resulting mature sperm must be able to fertilize.

With respect to the second hurdle, spermatogonial stem cells were recently cultured in vitro after which they proliferated for long periods of time in mice (16-18). Spermatogonial stem cells proliferated $10^{14}$-fold in a 5 -month period. In vitro culture may increase the rate of successful transplantation even with a small number of dissected seminiferous tubules. With respect to the third hurdle, a procedure for transplanting germ cells so that they undergo normal spermatogenesis has been established in animals $(8,9,19)$, and this normal spermatogenesis was further confirmed in our study. We too have reported normal spermatogenesis of transplanted germ cells $(13,14,20)$. We know the resulting mature sperm had the normal ability to fertilize because the rate of embryos reaching full term in this study $(28.6 \%)$ was the same as that of embryos constructed with normal sperm (21). The success of these studies in animals is encouraging with respect to potential application in the clinical setting. However, these procedures should be further refined before clinical application. The remaining problem in animal studies is the first one, exclusion of malignant cells from germ cells. Development of a procedure to isolate testicular germ cells from malignant cells and avoid contamination remains
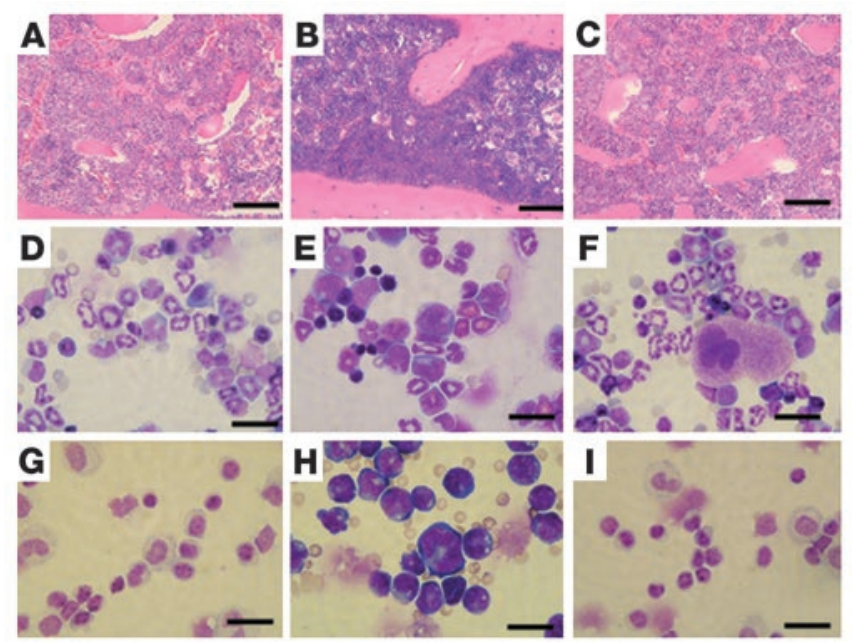


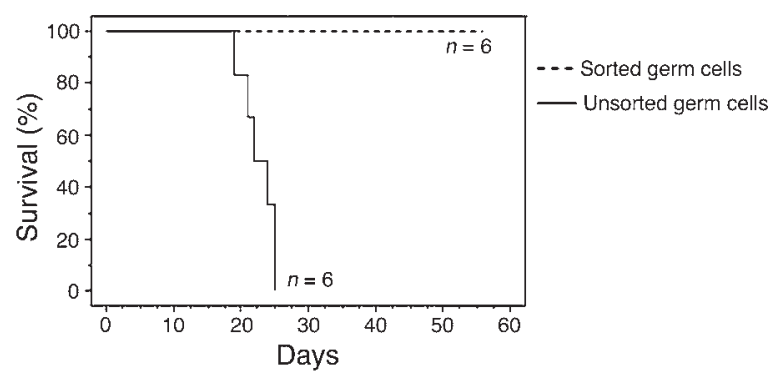

Figure 5

Survival after transplantation of sorted germ cells (dashed line, $n=6$ ) or unsorted germ cells (solid line, $n=6$ ) from C1498-inoculated GFP mice into seminiferous tubules. All mice receiving unsorted germ cells developed terminal signs of leukemia within 25 days.

one of the greatest challenges. In the present study, we successfully transplanted germ cells sorted from the testes of leukemic mice by staining them with surface markers and subjecting them to FACS. We also restored fertility in sterile mice exposed to busulfan, an alkylating agent used to treat chronic myeloid leukemia as well as in conditioning protocols before bone marrow transplantation for pediatric leukemia (22), without inducing leukemia.

Compared to using spermatids or spermatozoa from the testes of survivors, harvesting before treatment and autotransplantation has the advantage of preservation of fertility even in prepubertal patients who have no spermatids or spermatozoa. In addition, genetic damage of germ cells by chemotherapy and radiation therapy is prevented $(23,24)$. Difficulties include the need for critical assessment of the feasibility of the transplantation technique and of safety regarding normality of meiosis after cryopreserving and transplanting human stem cells (25). These techniques (for the autotransplantation of germ cells) have not been established in humans. In addition, ethical issues regarding patient autonomy and the need for informed consent should be addressed (26). Although dissection of testicular tissues from adult patients with azoospermia is performed safely (27), dissection of testicular tissues from prepubertal leukemic patients prior to treatment may increase risks of hemorrhage, infection, and deleterious gonadal function. Furthermore, more than half of the long-term survivors are not infertile and do not need to preserve spermatogonial stem cells $(3,4)$. Alkylating agents and radiation therapy for testes are high-risk factors for infertility $(4,28,29)$, but patients who need

\section{Figure 6}

Transplantation of germ cells derived from the testes of leukemic GFP mice into busulfan-treated tubules. (A and B) Cross-sections of a testis at 2 weeks after the transplantation of unsorted germ cells show infiltration by leukemic cells (H\&E). (C) Transplanted testis at 8 weeks after transplantation of the germ cell-enriched fraction sorted from the testes of leukemic GFP mice and (D) the corresponding fluorescence image. (E) Seminiferous tubules dissected from a transplanted testis and $(\mathbf{F})$ the corresponding fluorescence image. Donor germ cells (green) colonized in recipient seminiferous tubules. (G) Differentiated germ cells from a transplanted testis. (H and I) Cross-sections of a recipient testis transplanted with the sorted germ cell-enriched fraction. $(\mathbf{H})$ Hematoxylin stain. (I) Fluorescence image. Scale bars: $1 \mathrm{~mm}$ (A, C-F); $100 \mu \mathrm{m}$ (B, H, and I); $20 \mu \mathrm{m}$ (G). radiation therapy as a pretreatment for bone marrow transplantation have, among childhood leukemic patients, poor prognoses (30) and are in the minority of patients with childhood leukemia. This procedure should be strictly limited to candidates for preservation of spermatogonial stem cells. Finally, technical error may lead to contamination of donor cells by malignant cells.

With respect to potential for contamination, all 12 mice injected with the germ cell-enriched fractions (G2) sorted from leukemic mice survived for 300 days in this study. Histological examination of these mice showed no leukemia. Mice inoculated with 10 C1498 cells developed leukemia within 50 days (Figure 8). The kill curve for $\mathrm{C} 1498$ cells showed that 1 or 2 viable $\mathrm{C} 1498$ cells would kill mice in approximately 60 days (Figure 8 ). Therefore, it is reasonable to believe that all of the leukemic cells were excluded from the germ cell-enriched fraction. Mice inoculated with a single $\mathrm{C} 1498$ cell have been reported to develop leukemia (15), but 1 or 2 C1498 cells did not kill mice in our study. It is difficult to confirm the presence of 1 or 2 viable leukemic cells in an injection, and we cannot rule out the possibility that the germ cell-enriched fraction isolated from leukemic mice may have been contaminated with a few leukemic cells. To apply this procedure to the clinical setting, it is necessary to avoid transplanting as much as a single malignant cell, even if it is unlikely to proliferate and cause disease. Technical error may cause contamination of donor cells by malignant cells. Sensitive methods of detecting contamination by a single cell are therefore necessary. In vitro proliferation of spermatogonial stem cells may also expand contaminating leukemic cells in the same culture. Repeated isolation of germ cells combined with in vitro proliferation would reduce the risk of contamination by malignant cells. The germ cell-enriched fraction would be isolated from a testicular biopsy specimen and then cultured in vitro to expand the number of stem cells. Because spermatogonial stem cells proliferate, a single stem cell can form a colony (17), from which only 1 cell would be picked and proliferated in vitro. Theoretically, stem cells proliferated from 1 nonmalignant cell would contain no malignant cells. Only spermatogonial stem cells from mice have been proliferated in vitro to date; a procedure to proliferate human spermatogonial stem cells is necessary. A second FACS analysis for proliferated cells would have to be performed to confirm purity (31). No contamination by

\section{A}
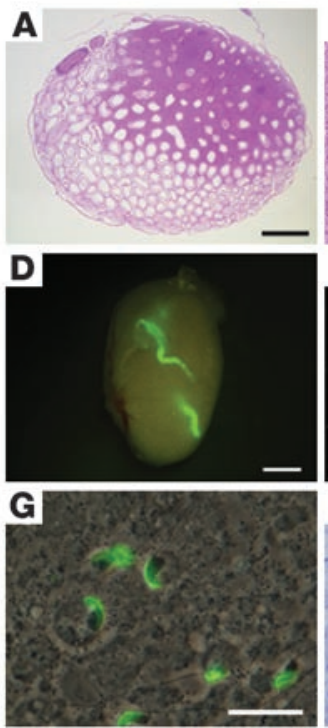
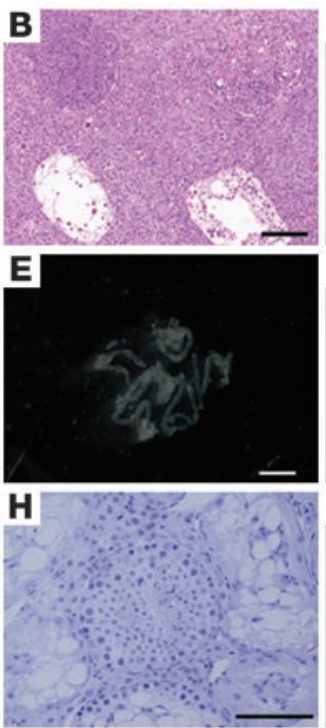
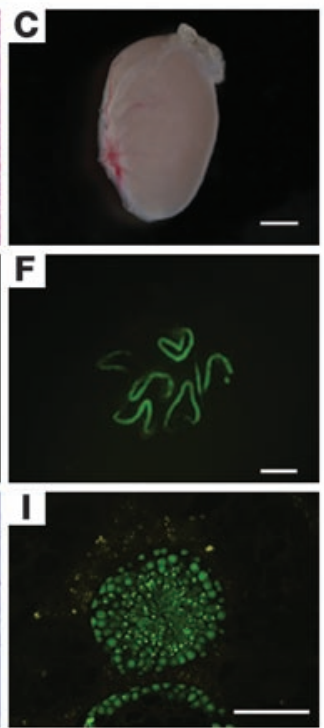

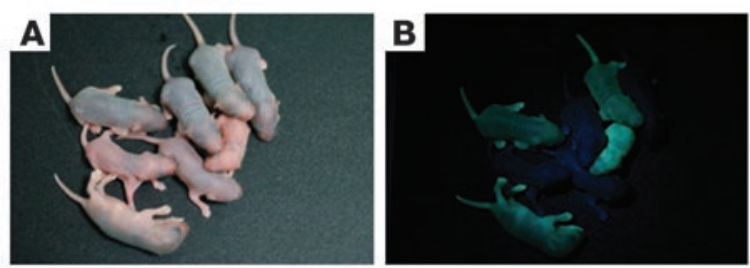

Figure 7

Sperm from transplanted testes support normal development. (A) Normal progeny produced by intracytoplasmic injection of GFP sperm obtained from transplanted recipient testes. (B) Corresponding fluorescence image. Because hemizygous GFP transgenic mice were used, approximately half of the pups showed green fluorescence.

malignant cells can also be confirmed by inoculation of a portion of donor cells into immunodeficient mice. A combination of germ cell isolation by FACS and in vitro proliferation would circumvent the problem of contamination by malignant cells.

Application of testis tissue xenografting for treating infertility in long-term survivors of childhood cancer has been proposed $(32,33)$. Xenografting of testis tissues from pigs and goats under the skin of immunodeficient mice has resulted in complete donor-derived spermatogenesis (33). The use of animals as recipients of testis grafts and the use of the resultant sperm for intracytoplasmic sperm injections eliminates the potential risk of transferring malignant cells to survivors because no tissue is transplanted to the survivors and only a mature sperm can be picked for intracytoplasmic sperm injections. However, because recipient mice are immunodeficient, contamination by malignant cells in grafted tissues may kill recipient mice before spermatid maturation occurs. Xenogeneic spermatogenesis also raises ethical issues and the risk of transmission of animal pathogens, particularly viruses, with the possible adaptation of such pathogens for human-to-human transmission (34). Finally, because infants conceived with the use of intracytoplasmic sperm injection or in vitro fertilization have a higher risk of major birth defects than do naturally conceived infants (35), autotransplantation of germ cells would be advantageous in that the donor can father a child by spontaneous conception (36).

To isolate germ cells from the testes of leukemic mice by FACS, surface markers are necessary because leukemic cells and germ cells cannot be differentiated by cell size, shape, or complexity. Many surface markers specific for spermatogonial stem cells have been reported $(37,38)$. Surface markers such as EE2, Thy-1.2, $\alpha 6$-integrin, $\beta 1$-integrin, EpCAM, and CD24 are expressed on spermatogonia or spermatogonial stem cells whereas c-kit, MHC-class I, and Sca-1 are not expressed on spermatogonial stem cells (37-40). For isolating germ cells, including stem cells, from leukemic cells, there is a need for surface markers that are expressed on germ cells but not on leukemic cells or a marker that is expressed on all leukemic cells. For example, germ cells expressing Thy-1.2 include enriched stem cells (37), but some C1498 cells also express Thy-1.2 (data not shown). Positive selection with the use of surface markers such as EE2 expressed only on germ cells risks contamination by leukemic cells because leukemic cells attach nonspecifically to germ cells and may be sorted into the germ cell-enriched fraction. Negative selection with the use of surface markers expressed only on leukemic cells is preferred because germ cells attached to leukemic cells are excluded by the surface markers expressed on leukemic cells. Leukemic cells express many surface markers, including CD45, CD13, and
CD69 $(41,42)$. We investigated the staining pattern of these antigens and selected 2 surface markers, CD45 and MHC class I, which are expressed on leukemic cells but not on germ cells. CD45 is expressed on all lymphohematopoietic cells: normal and malignant cells of the myeloid as well as T and B cell lineages (43). MHC class I is a heterodimer composed of a heavy chain and $\beta_{2}$-microglobulin, which is attached noncovalently to the heavy chain and is expressed on almost all somatic cells but not on testicular germ cells in humans and mice $(41,44,45)$. Both C57BL/6 mice and C1498 cells express $\mathrm{H}-2 \mathrm{~K}^{\mathrm{b}} /$ $\mathrm{H}-2 \mathrm{D}^{\mathrm{b}}$ as the MHC class I heavy chain. In the present study, MHC class I was used not only to exclude leukemic cells but also to enrich stem cells (37). Although we could not exclude all leukemic cells from the germ cell-enriched fractions by FACS sorting with 2 surface markers, $\mathrm{H}-2 \mathrm{~K}^{\mathrm{b}} / \mathrm{H}-2 \mathrm{D}^{\mathrm{b}}$ and $\mathrm{CD} 45$, the combination of surface markers with gating of the $\mathrm{FSC}^{\text {high }}$ and SSClow cell population made it possible to exclude all leukemic cells. The FSChigh and SSClow cell populations also possess spermatogonial stem cell activity (38). In this study, we selected antibodies against MHC class I and CD45 based on studies of the $\mathrm{C} 1498$ cell line. No cells from the mouse erythroleukemia cell line were found in the $\mathrm{H}-2 \mathrm{~K}^{\mathrm{b}} / \mathrm{H}-2 \mathrm{D}^{\mathrm{b}-}$ and $\mathrm{CD} 45^{-}$fractions. However, humans experience many types of leukemia. For example, approximately $10 \%$ of childhood acute lymphoblastic leukemias that are associated with a favorable prognosis are CD45-negative (46). Therefore, it is necessary to immunophenotype leukemic cells of individuals by flow cytometry or antibody microarray before isolation (47), and antibodies positive for leukemic cells in each patient should be used. The purity of the isolated germ cell-enriched fraction should be confirmed before transplantation by a second analysis with FACS or another sensitive method.

In conclusion, we isolated germ cells from leukemic mice by means of FACS and 2 surface markers, MHC-class I and CD45, and successfully transplanted germ cells into recipient testes. The lack of transmission of leukemia to recipient mice and the birth of normal offspring resulting from sorted germ cells show the potential of autotransplantation of germ cells isolated by FACS for the treatment of infertility secondary to anticancer treatment for childhood leukemia.

\section{Methods}

Preparation of donor leukemic mice and recipient mice. C1498, a murine leukemia cell line of C57BL/6 origin, was obtained from ATCC via Yoshihiro Oka (Osaka University, Osaka, Japan). Three-week-old C57BL/6 mice

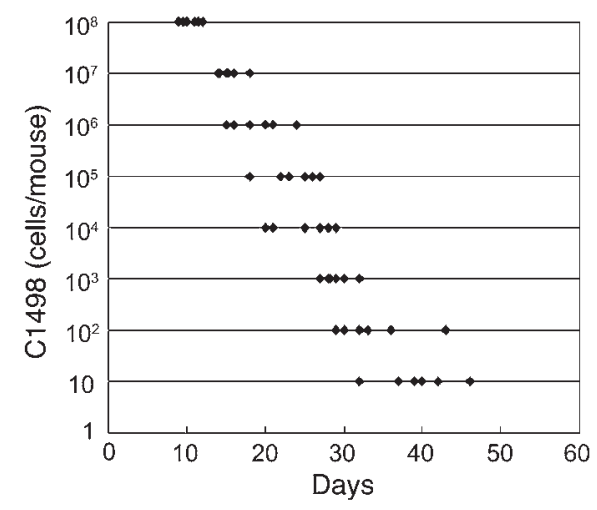

Figure 8

Kill curve of C1498 leukemia cells in mice. Six mice were injected with each indicated amount of leukemic cells, and the number of days of survival was plotted. 
were purchased from Shizuoka Laboratory Animal Center. C57BL/6 mice were inoculated intraperitoneally with $1 \times 10^{7} \mathrm{C} 1498$ cells in $200-\mu \mathrm{l}$ PBS. Two weeks after injection, 5-week-old mice with hemorrhagic ascites were killed, and both testes were removed. The inoculation dose of tumor cells was determined according to the kill curve so that mice reached the terminal state 2 weeks after inoculation (Figure 8). Six-week-old enhanced GFP (EGFP) transgenic mice (line no. C57BL/6TgN(acro/act-EGFP)OsbC3-N01FJ002; hereafter referred to as GFP mice) carrying both acrosin/EGFP (Acr3EGFP) and pCX-EGFP were used as donors of testicular germ cells $(20,48)$. GFP mice were also inoculated intraperitoneally with $1 \times 10^{7} \mathrm{C} 1498$ cells in 200- $\mu$ l PBS. Two weeks after injection, GFP mice with hemorrhagic ascites were killed, and both testes were removed. Four weeks prior to transplantation, 4-week-old recipient C57BL/6 mice were injected intraperitoneally with busulfan ( $40 \mathrm{mg} / \mathrm{kg}$; Sigma-Aldrich) dissolved in 50\% DMSO to eliminate endogenous spermatogenesis. All animal experiments were approved by the Animal Research Committee of Osaka University (Osaka, Japan) and were carried out in compliance with Osaka University's Guidelines on the Care and Use of Laboratory Animals.

Preparation of germ cells. Donor cells were prepared from the testes of normal 5-week-old C57BL/6 mice, 5-week-old C1498-inoculated C57BL/6 mice, and 8-week-old C1498-inoculated GFP mice. Briefly, after removal of the tunicae albugineae, the seminiferous tubules were placed in DMEM buffered with 20 mM HEPES at pH 7.3 and containing collagenase type IV $(1 \mathrm{mg} / \mathrm{ml})$ (Sigma-Aldrich), and the tubules were incubated for 15 minutes at $37^{\circ} \mathrm{C}$ with manual agitation for 5 minutes. The tubules were washed twice in calcium-free PBS and incubated in PBS containing 0.25\% trypsin and DNase I $(100 \mu \mathrm{g} / \mathrm{ml})$ (Sigma-Aldrich) for 15 minutes at $37^{\circ} \mathrm{C}$ with manual agitation at 5 -minute intervals. After the addition of a half-volume of DMEM containing $10 \%$ FBS, the cell suspension was filtered through $30-\mu \mathrm{m}$ pore size nylon mesh to remove large clumps of cells.

Cell immunostaining. Cell immunostaining was performed as described elsewhere (37). Dissociated germ cells were suspended in PBS containing $0.5 \%$ FBS (PBS/FBS). Cells were then incubated with biotin-conjugated anti-H-2 $\mathrm{K}^{\mathrm{b}} / \mathrm{H}-2 \mathrm{D}^{\mathrm{b}}$ (28-8-6; BD Biosciences - Pharmingen) for 20 minutes on ice and washed twice with excess PBS/FBS. Next, cells were incubated for 20 minutes with PE-conjugated streptavidin (BD Biosciences - Pharmingen) and either FITC-conjugated anti-CD45 (30-F11; BD Biosciences - Pharmingen) for FACS sorting of non-GFP germ cells or PE-Cy5-conjugated anti-CD45 (30-F11; BD Biosciences - Pharmingen) for FACS sorting of GFP cells. All primary antibodies and secondary reagents were used at a concentration of $5 \mu \mathrm{g} / \mathrm{ml}$. C1498 cells were stained with these antibodies as a positive control. After a final wash, cells were resuspended $\left(4 \times 10^{6}\right.$ cells $/ \mathrm{ml}$ ) in PBS/FBS containing $1 \mu \mathrm{g} / \mathrm{ml}$ propidium iodide (PI) (SigmaAldrich) and kept in the dark on ice until FACS sorting.

FACS. Cell sorting was performed with a FACSVantage Cell Sorter (BD Biosciences) equipped with a $488-\mathrm{nm}$ argon laser $(250 \mathrm{~mW})$. The laser was used to excite FITC, PE, PI, and PE-Cy5, and fluorescence emissions were collected with a 530-nm filter for FITC, a 575-nm filter for PE and PI, and a 682-nm filter for PE-Cy5 and PI. The gate (G1) was set on FSC versus SSC parameters. Cells in the germ cell-enriched fraction (G2) were sorted by negative labeling for $\mathrm{H}-2 \mathrm{~K}^{\mathrm{b}} / \mathrm{H}-2 \mathrm{D}^{\mathrm{b}}$-streptoavidin PE, PI, and CD45 FITC/ $\mathrm{PE}-\mathrm{Cy} 5$, and cells in the leukemic cell fraction (G3) were sorted by positive labeling for $\mathrm{H}-2 \mathrm{~K}^{\mathrm{b}} / \mathrm{H}-2 \mathrm{D}^{\mathrm{b}}$-streptoavidin PE and CD 45 FITC/PE-Cy5. Data for 10,000 events were analyzed with CELLQuest software version 3.3 (BD Biosciences). Cells were sorted into 5-ml tubes containing 2-ml DMEM supplemented with $10 \%$ FBS (DMEM/FBS). The FSC threshold was set to exclude cell debris. Viability of isolated cells was assessed by trypan blue exclusion. The sorted cells were collected, centrifuged, and resuspended in $10-\mathrm{ml}$ cold DMEM/FBS. The tube was gassed with $5 \% \mathrm{CO}_{2}$ and stored overnight at $4^{\circ} \mathrm{C}$.
Immunocytochemistry of germ cells. Dissociated germ cells from leukemic mice and the germ cell-enriched fraction isolated from leukemic mice were cytospun onto glass slides ( 5 minutes at $28 \mathrm{~g}$; Cytospin 3 , Shandon Inc.). Cytospin preparations were fixed in 100\% ethanol for 15 minutes and washed with PBS, and endogenous peroxidase activity was blocked with 3\% hydrogen peroxide. The sections were incubated with TRA98 (a gift from Yoshitake Nishimune and Hiromitsu Tanaka, Osaka University, Osaka, Japan) at a dilution of 1:1000 for 1 hour at $37^{\circ} \mathrm{C}$. After washing and incubation with peroxidase-conjugated goat anti-rat IgG antibody (ICN Pharmaceuticals Inc.) for 60 minutes at room temperature at a dilution of 1:1000, the slides were treated with diaminobenzidine in PBS, counterstained with hematoxylin, dehydrated, and mounted.

Intraperitoneal injection. A total of $2 \times 10^{6}$ cells of the germ cell-enriched fraction and $1 \times 10^{5}$ cells of the leukemic cell fraction sorted from testes of C1498-inoculated C57BL/ 6 mice were suspended in 100- $\mu$ l PBS/FCS. The cell suspension was injected intraperitoneally into C57BL/6 mice. The mice were killed when they approached the terminal phase, characterized by the presence of hemorrhagic ascites, and bone marrow from a femoral bone and ascites were cytospun onto glass slides ( 5 minutes, $55 \mathrm{~g}$ ) and stained with May-Giemsa stain. Another femoral bone was fixed in $4 \%$ paraformaldehyde, decalcified, embedded in paraffin, cut into $5-\mu \mathrm{m}$-thick sections, and stained with $\mathrm{H} \& \mathrm{E}$.

Transplantation of germ cells from C1498-inoculated GFP mice. Dissociated testicular cells from C1498-inoculated GFP mice were sorted by FACS with $\mathrm{H}-2 \mathrm{~K}^{\mathrm{b}} / \mathrm{H}-2 \mathrm{D}^{\mathrm{b}}$-streptavidin PE, PI, and CD45 PE-Cy5 as described above. The sorted germ cell-enriched fraction was washed with injection medium (138 mM NaCl, $8.1 \mathrm{mM} \mathrm{Na}_{2} \mathrm{HPO}_{4}, 2.7 \mathrm{mM} \mathrm{KCl}, 1.1 \mathrm{mM} \mathrm{KH}_{2} \mathrm{PO}_{4}, 0.1 \mathrm{mM}$ EDTA, $5.5 \mathrm{mM}$ glucose, $5 \mathrm{mg} / \mathrm{ml} \mathrm{BSA}, 100 \mu \mathrm{g} / \mathrm{ml}$ DNase I, and $0.4 \mathrm{mg} /$ $\mathrm{ml}$ trypan blue) (8) and suspended in injection medium at a concentration of $1 \times 10^{8}$ cells $/ \mathrm{ml}$. Transplantation of donor cells into seminiferous tubules was performed via injection into the efferent ductules 4 weeks after busulfan treatment (20). Approximately $10 \mu \mathrm{l}$ of donor cell suspension was introduced per testis. Transplantation of unsorted germ cells dissociated from C1498-inoculated GFP mice was also performed as described above. Mice were killed at the onset of the terminal phase or at 8 weeks after transplantation, and the testes were analyzed. Testes transplanted with unsorted germ cells at 2 weeks after transplantation or testes from mice with hemorrhagic ascites were fixed in Bouin's solution, embedded in paraffin, cut into 5 - $\mu \mathrm{m}$-thick sections, and stained with H\&E. Otherwise, testes were exposed to excitation by ultraviolet light and photographed with an Olympus SZX-12 camera. In order to enable detailed observation of colonized GFP germ cells, the recipient testes were fixed in $4 \%$ paraformaldehyde at $4^{\circ} \mathrm{C}$ overnight and embedded in glycol methacrylate (Technovit 8100; Kulzer), and 5 - $\mu \mathrm{m}$-thick serial sections were prepared at 50- $\mu \mathrm{m}$ intervals. After observation of GFP fluorescence, the sections were stained with hematoxylin and observed under a photomicroscope.

Intracytoplasmic sperm injection. Testicular sperm were prepared from the recipient testes at 8 weeks after transplantation. Briefly, seminiferous tubules with green fluorescence were collected from recipient testes under a fluorescence stereomicroscope, minced with fine scissors, and suspended in Hepes-Chatot-Ziomek-Bavister (Hepes-CZB) medium (49). Mature oocytes were collected as follows. BDF1 female mice were induced to superovulate by intraperitoneal injection of $5 \mathrm{IU}$ pregnant mare serum gonadotropin (Teikoku Hormone Manufacturing Co.) followed 48 hours later by injection of 5 IU human chorionic gonadotropin (hCG) (Teikoku Hormone Manufacturing Co.). Mature oocytes were collected from the ampullary region of the oviducts $13-15$ hours after hCG injection. Oocytes were freed from cumulus cells by treatment with $0.1 \%$ bovine testicular hyaluronidase (Sigma-Aldrich) in Hepes-CZB medium. Oocytes were rinsed and kept in fresh $\mathrm{CZB}$ medium at $37^{\circ} \mathrm{C}$ and $5 \% \mathrm{CO}_{2}$ until sperm injection. Approximately $2 \mu \mathrm{l}$ of the GFP sperm suspension collected from 
recipient testes was mixed with a drop of Hepes-CZB medium containing $12 \%(\mathrm{w} / \mathrm{v})$ polyvinylpyrrolidone (PVP) $\left(\mathrm{M}_{\mathrm{r}} 360,000\right.$; Wako Pure Chemical Industries). Sperm heads were separated from tails by application of a few piezo pulses to the sperm neck region and were injected into oocytes as described elsewhere (21). Oocytes were incubated in CZB medium at $37^{\circ} \mathrm{C}$ and $5 \% \mathrm{CO}_{2}$ in air. Two-cell embryos were transferred into the oviducts of 0.5 -dpc pseudopregnant ICR females.

\section{Acknowledgments}

We thank Yoshihiro Oka, Hiroko Nakajima, and Sumiyuki Nishida for advice regarding the C1498 cell line; Yoshitake Nishimune and Hiromitsu Tanaka for kindly providing the TRA98 antibody; Masaru Okabe for kindly providing GFP mice; and Hiroshi Yamazaki for technical advice regarding FACS.

Received for publication December 14, 2004, and accepted in revised form May 10, 2005.

Address correspondence to: Akira Tsujimura, Osaka University Graduate School of Medicine, 2-2 Yamadaoka, Suita Osaka 5650871, Japan. Phone: 81-6-6879-3531; Fax: 81-6-6879-3539; E-mail: akitsuji@uro.med.osaka-u.ac.jp.
1. U.S. Cancer Statistics Working Group. 2004 United States cancer statistics: 2000 incidence and mortality - updated and enhanced. Department of Health and Human Services, Centers for Disease Control and Prevention, and National Cancer Institute. Atlanta, Georgia, USA. http://apps.nccd. cdc.gov/uscs/index.asp?Year=2000.

2. Jemal, A., et al. 2004. Annual report to the nation on the status of cancer, 1975-2001, with a special feature regarding survival. Cancer. 101:3-27.

3. Humpl, T., Schramm, P., and Gutjahr, P. 1999. Male fertility in long-term survivors of childhood ALL. Arch. Androl. 43:123-129.

4. Thomson, A.B., et al. 2002. Semen quality and spermatozoal DNA integrity in survivors of childhood cancer: a case-control study. Lancet. 360:361-367.

5. Bauld, C., Anderson, V., and Arnold, J. 1998. Psychosocial aspects of adolescent cancer survival. J. Paediatr. Child Health. 34:120-126.

6. Agarwal, A., et al. 2004. Fertility after cancer: a prospective review of assisted reproductive outcome with banked semen specimens. Fertil. Steril. 81:342-348.

7. Hellstrom, W.J.G. 1997. Male infertility and sexual dysfunction. Springer. New York, New York, USA. 606 pp.

8. Brinster, R.L., and Zimmermann, J.W. 1994. Spermatogenesis following male germ-cell transplantation. Proc. Natl. Acad. Sci. U. S. A. 91:11298-11302.

9. Brinster, R.L., and Avarbock, M.R. 1994. Germline transmission of donor haplotype following spermatogonial transplantation. Proc. Natl. Acad. Sci. U. S. A. 91:11303-11307.

10. Schlatt, S., Foppiani, L., Rolf, C., Weinbauer, G.F., and Nieschlag, E. 2002. Germ cell transplantation into X-irradiated monkey testes. Hum. Reprod. 17:55-62.

11. Jahnukainen, K., Hou, M., Petersen, C., Setchell, B. and Soder, O. 2001. Intratesticular transplantation of testicular cells from leukemic rats causes transmission of leukemia. Cancer Res. 61:706-710.

12. Tanaka, H., et al. 1997. A germ cell-specific nuclear antigen recognized by a monoclonal antibody raised against mouse testicular germ cells. Int. J. Androl. 20:361-366.

13. Ohta, H., et al. 2001. Defect in germ cells, not in supporting cells, is the cause of male infertility in the jsd mutant mouse: proliferation of spermatogonial stem cells without differentiation. Int. J. Androl. 24:15-23.

14. Ohta, H., Yomogida, K., Dohmae, K., and Nishimune, Y. 2000. Regulation of proliferation and differentiation in spermatogonial stem cells: the role of c-kit and its ligand SCF. Development. 127:2125-2131.

15. Goldie, H., Butler, C.H., Anderson, M.M., Maxwell, M.C., and Hahn, P.F. 1953. Growth characteristics of free C1498 granulocytic leukemia tumor cells in the peritoneal fluid and the blood of C57 mice. Cancer Res. 13:125-129.

16. Nagano, M., Ryu, B.Y., Brinster, C.J., Avarbock, M.R., and Brinster, R.L. 2003. Maintenance of mouse male germ line stem cells in vitro. Biol. Reprod. 68:2207-2214.

17. Kanatsu-Shinohara, M., et al. 2003. Long-term proliferation in culture and germline transmission of mouse male germline stem cells. Biol. Reprod. 69:612-616

18. Kubota, H., Avarbock, M.R., and Brinster, R.L. 2004. Growth factors essential for self-renewal and expansion of mouse spermatogonial stem cells. Proc. Natl. Acad. Sci. U. S. A. 101:16489-16494.

19. Kanatsu-Shinohara, M., et al. 2003. Restoration of fertility in infertile mice by transplantation of cryopreserved male germline stem cells. Hum. Reprod. 18:2660-2667.

20. Ohta, H., Yomogida, K., Yamada, S., Okabe, M., and Nishimune, Y. 2000. Real-time observation of transplanted 'green germ cells': proliferation and differentiation of stem cells. Dev. Growth Differ. 42:105-112.

21. Kimura, Y., and Yanagimachi, R. 1995. Intracytoplasmic sperm injection in the mouse. Biol. Reprod. 52:709-720.

22. Shah, A.J., et al. 2004. Busulfan and cyclophosphamide as a conditioning regimen for pediatric acute lymphoblastic leukemia patients undergoing bone marrow transplantation. J. Pediatr. Hematol. Oncol. 26:91-97.

23. Robbins, W.A., et al. 1997. Chemotherapy induces transient sex chromosomal and autosomal aneuploidy in human sperm. Nat. Genet. 16:74-78.

24. Brandriff, B.F., Meistrich, M.L., Gordon, L.A., Carrano, A.V., and Liang, J.C. 1994. Chromosomal damage in sperm of patients surviving Hodgkin's disease following MOPP (nitrogen mustard, vincristine, procarbazine, and prednisone) therapy with and without radiotherapy. Hum. Genet. 93:295-299.

25. Tournaye, H., et al. 2004. Preserving the reproductive potential of men and boys with cancer: current concepts and future prospects. Hum. Reprod. Update. 10:525-532.

26. Bahadur, G. 2004. Ethics of testicular stem cell medicine. Hum. Reprod. 19:2702-2710.

27. Komori, K., et al. 2004. Serial follow-up study of serum testosterone and antisperm antibodies in patients with non-obstructive azoospermia after conventional or microdissection testicular sperm extraction. Int. J. Androl. 27:32-36.

28. Sklar, C.A., et al. 1990. Effects of radiation on testicular function in long-term survivors of childhood acute lymphoblastic leukemia: a report from the Children Cancer Study Group. J. Clin. Oncol. 8:1981-1987.

29. Wallace, W.H., Shalet, S.M., Lendon, M., and Morris-Jones, P.H. 1991. Male fertility in long-term survivors of childhood acute lymphoblastic leukaemia. Int. J. Androl. 14:312-319.

30. Arico, M., et al. 2000. Outcome of treatment in children with Philadelphia chromosome-positive acute lymphoblastic leukemia. N. Engl. J. Med. 342:998-1006.

31. Campana, D., and Coustan-Smith, E. 1999. Detection of minimal residual disease in acute leukemia by flow cytometry. Cytometry. 38:139-152.

32. Aslam, I., Fishel, S., Moore, H., Dowell, K., and Thornton, S. 2000. Fertility preservation of boys undergoing anti-cancer therapy: a review of the existing situation and prospects for the future. Hum. Reprod. 15:2154-2159.

33. Honaramooz, A., et al. 2002. Sperm from neo- natal mammalian testes grafted in mice. Nature. 418:778-781.

34. Boneva, R.S., and Folks, T.M. 2004. Xenotransplantation and risks of zoonotic infections. Ann. Med. 36:504-517.

35. Hansen, M., Kurinczuk, J.J., Bower, C., and Webb, S. 2002. The risk of major birth defects after intracytoplasmic sperm injection and in vitro fertilization. N. Engl. J. Med. 346:725-730.

36. Ogawa, T., Dobrinski, I., Avarbock, M.R., and Brinster, R.L. 2000. Transplantation of male germ line stem cells restores fertility in infertile mice. Nat. Med. 6:29-34.

37. Kubota, H., Avarbock, M.R., and Brinster, R.L. 2003. Spermatogonial stem cells share some, but not all, phenotypic and functional characteristics with other stem cells. Proc. Natl. Acad. Sci. U. S. A. 100:6487-6492.

38. Shinohara, T., Orwig, K.E., Avarbock, M.R., and Brinster, R.L. 2000. Spermatogonial stem cell enrichment by multiparameter selection of mouse testis cells. Proc. Natl. Acad. Sci. U. S. A. 97:8346-8351.

39. Koshimizu, U., Nishioka, H., Watanabe, D., Dohmae, K., and Nishimune, Y. 1995. Characterization of a novel spermatogenic cell antigen specific for early stages of germ cells in mouse testis. Mol. Reprod. Dev. 40:221-227.

40. Moore, T.J., de Boer-Brouwer, M., and van DisselEmiliani, F.M. 2002. Purified gonocytes from the neonatal rat form foci of proliferating germ cells in vitro. Endocrinology. 143:3171-3174.

41. Hotta, C., et al. 2000. Impaired expression of MHC class I molecules on mouse testicular germ cells is mainly caused by the post-transcriptional mechanism. Immunogenetics. 51:624-631.

42. LaBelle, J.L., and Truitt, R.L. 2002. Characterization of a murine NKT cell tumor previously described as an acute myelogenous leukemia. Leuk. Lymphoma. 43:1637-1644.

43. Hendrickx, A., and Bossuyt, X. 2001. Quantification of the leukocyte common antigen (CD45) in mature B-cell malignancies. Cytometry. 46:336-339.

44. Guillaudeux, T., et al. 1996. Expression of HLA class I genes in meiotic and post-meiotic human spermatogenic cells. Biol. Reprod. 55:99-110.

45. Kurpisz, M., and Fernandez, N. 1995. Immunology of buman reproduction. Bios Scientific. Oxford, United Kingdom. 509 pp.

46. Ratei, R, et al. 1998. Immunophenotype and clinical characteristics of CD45-negative and CD45positive childhood acute lymphoblastic leukemia. Ann. Hematol. 77:107-114.

47. Belov, L., de la Vega, O., dos Remedios, C.G., Mulligan, S.P., and Christopherson, R.I. 2001. Immunophenotyping of leukemias using a cluster of differentiation antibody microarray. Cancer Res. 61:4483-4489.

48. Nakanishi, T., et al. 1999. Real-time observation of acrosomal dispersal from mouse sperm using GFP as a marker protein. FEBS Lett. 449:277-283.

49. Chatot, C.L., Ziomek, C.A., Bavister, B.D., Lewis, J.L., and Torres, I. 1989. An improved culture medium supports development of random-bred 1-cell mouse embryos in vitro. J. Reprod. Fertil. 86:679-688. 